\title{
Pathologic interpretation of endoscopic ultrasound-guided fine needle aspiration cytology/biopsy for pancreatic lesions
}

\author{
Haeryoung Kim ${ }^{1}$, Kee-Taek Jang ${ }^{2}$ \\ ${ }^{1}$ Department of Pathology, Seoul National University Hospital, Seoul National University College of Medicine, Seoul; \\ 2Department of Pathology and Translational Genomics, Samsung Medical Center, Sungkyunkwan University School of Medicine, Seoul, Korea
}

\begin{abstract}
Pathologic interpretation of endoscopic ultrasound-guided fine needle aspiration (EUS-FNA) cytology/biopsy specimens is one of the most challenging tasks in cytology and surgical pathology practice, as the procedure often yields minimal amounts of diagnostic material and contains contaminants, such as blood cells and normal intestinal mucosa. EUS-FNA cytology/biopsy will nevertheless become a more popular procedure for evaluation of various pancreatic lesions because they are difficult to approach with conventional endoscopic procedures. Pathologists should understand the structural differences and limitations of EUS-FNA that make pathologic diagnosis difficult. Ancillary tests are available for differential diagnosis of EUS-FNA for various pancreatic lesions. Immunostains are the most commonly used ancillary tests, and pathologists should able to choose the necessary panel for differential diagnosis. Pathologists should review clinical history and radiologic and/or EUS findings before selecting an immunostain panel and making a pathologic diagnosis. In addition, one's threshold of malignancy should be adjusted according to the appropriate clinical setting to avoid under-evaluation of pathologic diagnoses. Clinico-pathologic correlation is essential in pathologic evaluation of EUS-FNA for pancreatic lesions. Pathologists can reduce errors by correlating clinical and radiologic findings when evaluating EUS-FNA. Some molecular tests can be applied in differential diagnosis of pancreatic neoplastic and cystic lesions. Molecular data should be used as supportive evidence of a specific disease entity, rather than direct evidence, and should be correlated with clinico-pathologic findings to avoid errors in pathologic diagnosis.
\end{abstract}

Key Words: Endoscopic ultrasound-guided fine needle aspiration; Pancreatic neoplasms; Pathology

Received: June 9, 2020 Revised: July 5, 2020 Accepted: July 21, 2020

Corresponding Author: Kee-Taek Jang, MD, Department of Pathology and Translational Genomics, Samsung Medical Center, Sungkyunkwan University School of Medicine, 81 Irwon-ro, Gangnam-gu, Seoul 06351, Korea

Tel: +82-2-3410-2763, Fax: +82-2-3410-0025, E-mail: ktjang12@gmail.com

Endoscopic ultrasound-guided fine needle aspiration (EUSFNA) cytology/biopsy is currently performed at many institutions and has become a routine procedure for pathologic diagnosis of pancreatic lesions. Although endoscopic ultrasound scanning began in the early 1980s [1], it has gained widespread popularity since the introduction of fine needle aspiration cytology/biopsy devices, which allow guided biopsies of target lesions visualized by endoscopic ultrasound [2]. Before the era of EUSFNA, it was difficult to target pancreatic lesions due to limited accessibility by percutaneous needle biopsies. Hence, pathologists could rarely encounter cytology and/or biopsy material from pancreatic lesions. With the increasing popularity of EUS-FNA procedures, interpretation of EUS-FNA cytology/biopsy material has become an almost inevitable part of routine practice for pathologic diagnosis of various pancreatic lesions. A main con- cern of pancreatic EUS-FNA specimen interpretation is the limited amount of aspirated material. Compared to aspirated material from superficial organs, such as the thyroid, breast, or uterine cervix, EUS-FNA from pancreatic lesions provides aspirates with relatively limited cellularity that may seem inadequate to less experienced pathologists. However, EUS-FNA inevitably results in lower cellularity compared to aspirates from other superficial organs. To make an appropriate diagnosis, pathologists should consider the clinical impression, especially radiologic and/or EUS findings; otherwise, many EUS-FNA specimens may be interpreted as "inadequate specimen". It is occasionally necessary to deem the sample "inadequate", "atypical", or of "uncertain malignant potential", which are diagnostically less useful to clinicians, but necessary. The use of these indeterminate categories will be reduced with expertise and correlation of cytologic 
findings with the clinico-radiological findings. If these categories are overused, communication and trust between clinicians and pathologists may become compromised. Pathologists may require time and experience to adjust to the relatively paucicellular smears of EUS-FNA specimens and their potential artifacts, including contaminants. In this review, we summarize the key cytologic findings of common pancreatic lesions, review preanalytic parameters that may affect the pathologic diagnosis of EUS-FNA, and tips for clinicopathologic correlation that may be helpful in the differential diagnosis of EUS-FNA for various pancreatic lesions.

\section{DIAGNOSTIC CONSIDERATIONS}

\section{Different preparation methods}

In most institutions, pancreatic EUS-FNA aspirates are prepared for cytologic review by the direct smear method. Direct smears are usually performed in the endoscopy suite; as soon as the aspirated material is obtained, the material is expelled onto glass slides. Grossly visible solid particles are placed into formalin for histopathologic examination, while the remaining material is smeared on site. The smears are either placed directly into alcohol fixative for Papanicolaou or hematoxylin-eosin stain or air-dried for subsequent Diff-Quik stain. If smeared properly without crushing or air dry artifacts, Papanicolaou or hematoxylin-eosin stains result in smears with high nuclear detail, which is important for pathologic diagnosis of various pancreatic neoplasms. Direct feedback helps to improve cytology smears, including microscopic observation of the smear slide by the person who actually performs the smear. Unfortunately, cytology smear preparation and cytology interpretation are usually performed by different people. Romanowsky stains, such as DiffQuik, are useful for analyzing background material, such as mucin, and for examining cytoplasmic features. Cell blocks, which may be useful for ancillary immunohistochemical stains, may also be prepared from aspirates. Recently, liquid-based cytology (LBC; e.g. ThinPrep, SurePath) has also been used for pancreatic EUS-FNA cytology.

\section{Liquid-based cytology}

LBC has become a standard method for cytologic evaluation of some organs, including the uterine cervix and thyroid. LBC can eliminate unnecessary background inflammatory and blood cells to increase diagnostic accuracy. Recent studies reported that LBC showed similar results to conventional smear for diagnosis of pancreatic masses $[3,4]$. The use of LBC may be limited for mucinous neoplasms because mucin is more dilute and scant on LBC preparations.

\section{Contaminants}

Because EUS-FNA involves penetration of either the gastric or duodenal wall to access the pancreas, most EUS-FNA aspirates contain at least a small amount of normal gastrointestinal mucosa. This is a very important pitfall in the interpretation of pancreatic EUS-FNA specimens, as normal gastrointestinal contaminants may be mistaken for neoplastic epithelial cells. Gastric foveolar epithelium appears as large irregular folded or monolayered sheets of mucin-containing columnar cells (Fig. 1A). The columnar cells have basally oriented nuclei with a palisaded appearance, and the luminal border is frequently seen
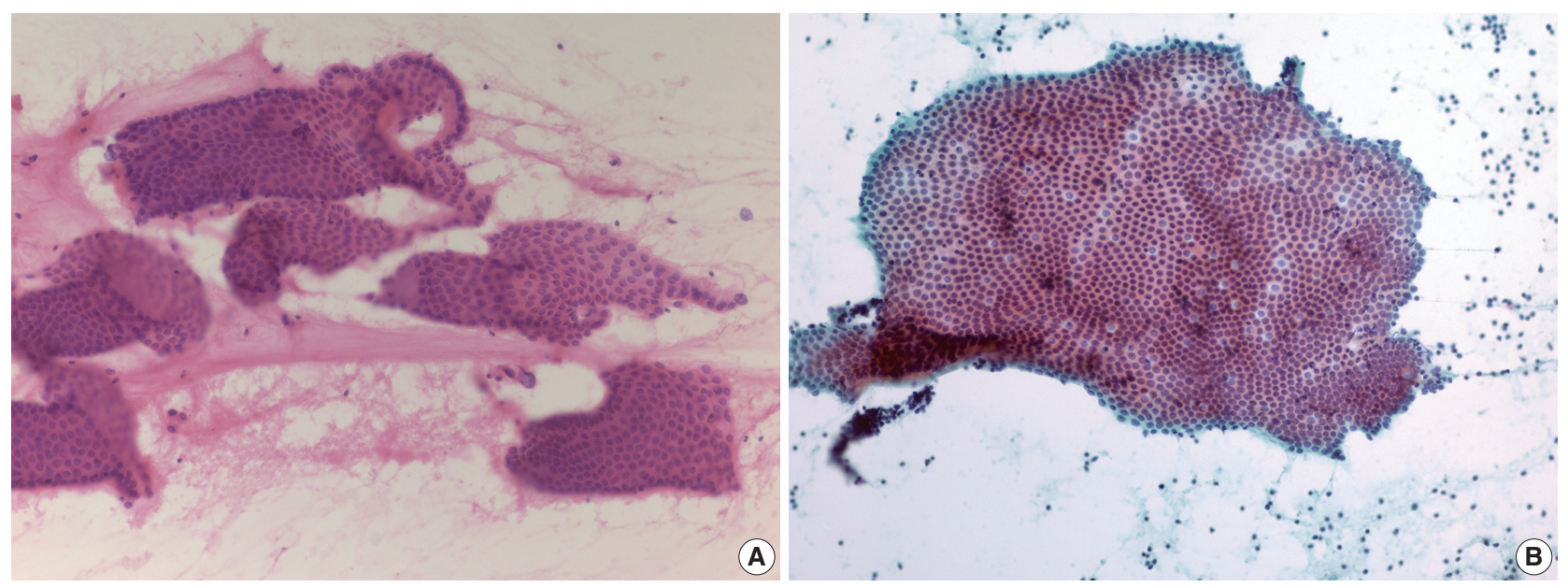

Fig. 1. (A). Normal gastric mucosa in conventional cytology smear shows nests of monotonous cells with smooth boundaries. (B) Normal duodenal mucosa showing scattered goblet cells in conventional cytology smear (Pap smear). 
along one edge of the epithelial cell sheets. Duodenal epithelium is also characterized by sheets of columnar cells with interspersed goblet cells, resulting in a characteristic "starry-sky" appearance (Fig. 1B). However, the most common contaminants are red blood cells, which obscure the cytologic feature of EUSFNA aspirates. Cell blocks are alternative tools to help overcome bloody smears; however, the efficiency of conventional cell block preparation is sometimes suboptimal. The tissue coagulum clot method reportedly increases cell block cellularity with endobronchial ultrasound-guided transbronchial fine-needle aspiration [5]. However, more validation studies are needed to apply this method to EUS-FNA for pancreatic lesions.

\section{Interpretation of pancreatic cystic lesions}

As cystic lesions comprise 2\%-13\% of pancreatic lesions [6], it is not uncommon for pathologists to encounter EUS-FNA specimens for pancreatic cystic lesions. While characteristic cytologic features may help in diagnosis when a mural or papillary nodule is present within the pancreatic cystic lesion, purely cystic lesions without mural or papillary nodules may yield paucicellular smears or only scattered macrophages. These cases would be interpreted as "inadequate" according to ordinary criteria for cytology diagnosis. However, it is difficult to obtain adequate cellularity from these cystic lesions despite repeated procedures. This is especially true for unilocular cystic lesions, such as pseudocysts, macrocystic serous or mucinous cystadenomas, and cystically dilated intraductal papillary mucinous neoplasms (IPMNs) without mural nodules; if the gross and microscopic findings of these cystic lesions are considered, it is logical that not enough cells or tissue may be obtained by the EUS-FNA procedure. In this situation, it may be more reasonable to interpret the findings as "benign cystic lesion" than "inadequate specimen." Although a hidden malignancy may exist around the "benign cystic lesion", this pattern is very rarely identified. In daily practice, benign cystic lesions, such as serous or mucinous cystadenomas, cystic IPMNs, and pseudocysts, are more common. Pathologists should correlate radiologic and EUS findings to avoid underevaluation of pathologic diagnosis for these cystic lesions. If imaging and EUS findings are concordant, it is not difficult to predict the biologic behavior of pancreatic cystic lesions. However, discrepancy between imaging and EUS interpretation for these cystic lesions is not uncommon. In this scenario, pathologists should raise the threshold for malignancy in pathologic diagnosis of EUS-FNA. Clinico-pathologic correlation is important for adequate pathologic interpretation of EUS-FNA from various pancreatic lesions.

\section{KEY PATHOLOGIC FEATURES OF COMMONLY ENCOUNTERED PANCREATIC NEOPLASMS}

\section{Ductal adenocarcinoma}

Pancreatic ductal adenocarcinoma is the most common solid tumor of the pancreas and the most commonly encountered pancreatic neoplasm on EUS-FNA. Aspirated material usually demonstrates atypical cells with or without a necrotic background. The cellularity of the cytologic smear depends on many parameters, including tumor size, location, cellularity, type of aspiration needle, and endoscopist technique [7-9]. A pathologist is more likely to encounter a paucicellular smear than a highly cellular smear. If many atypical cells are found in a necrotic background (Fig. 2A), it is easy to conclusively diagnose a malignancy even without clinical information. However, pathologists may encounter paucicellular smears showing only a few atypical cells without a necrotic background. In such situations, it is difficult to differentiate reactive ductal cell atypia of chronic pancreatitis from malignancy. Pathologists should correlate with the clinical impression and radiologic findings of these pancreatic lesions. If the clinical finding impression is an overt malignancy, such as pancreatic tumor with vascular encasement or liver metastasis, the pathologic diagnosis of malignancy can be made with a very limited volume of atypical cells. Pancreatic ductal adenocarcinomas are often well-differentiated with very mild cytological atypia, and one should be careful not to mistake them for contaminated gastric foveolar epithelium. In such cases, the architecture of cell groups should be observed in detail, including cribriforming, nuclear stratification, and loss of regular honeycombing seen in normal and benign epithelia (“drunken honeycomb”). Well differentiated tumor cell nests usually exhibit more cellularity with overlapping and touching nuclei (Fig. 2B). The presence of scattered small clusters of atypical cells with necrotic cell debris in the background is also helpful, as pancreatic ductal adenocarcinomas frequently demonstrate necrotic backgrounds and scattered small atypical cell nests or clusters. However, this necrotic background should be discriminated from the fibrin clots that are frequently observed in bloody smears (Fig. 2C). Another EUS-FNA specimen that demonstrates diffuse necrosis is tuberculous lymphadenitis involving peripancreatic or retroperitoneal lymph nodes: if the EUS-FNA cytology/biopsy shows a diffusely necrotic background without apparently viable tumor cells (Fig. 2D), the pathologist should check the clinical impression and imaging findings. In many cases, the radiologic impression is lymphoma, especially if there are multiple enlarged lymph nodes around the 

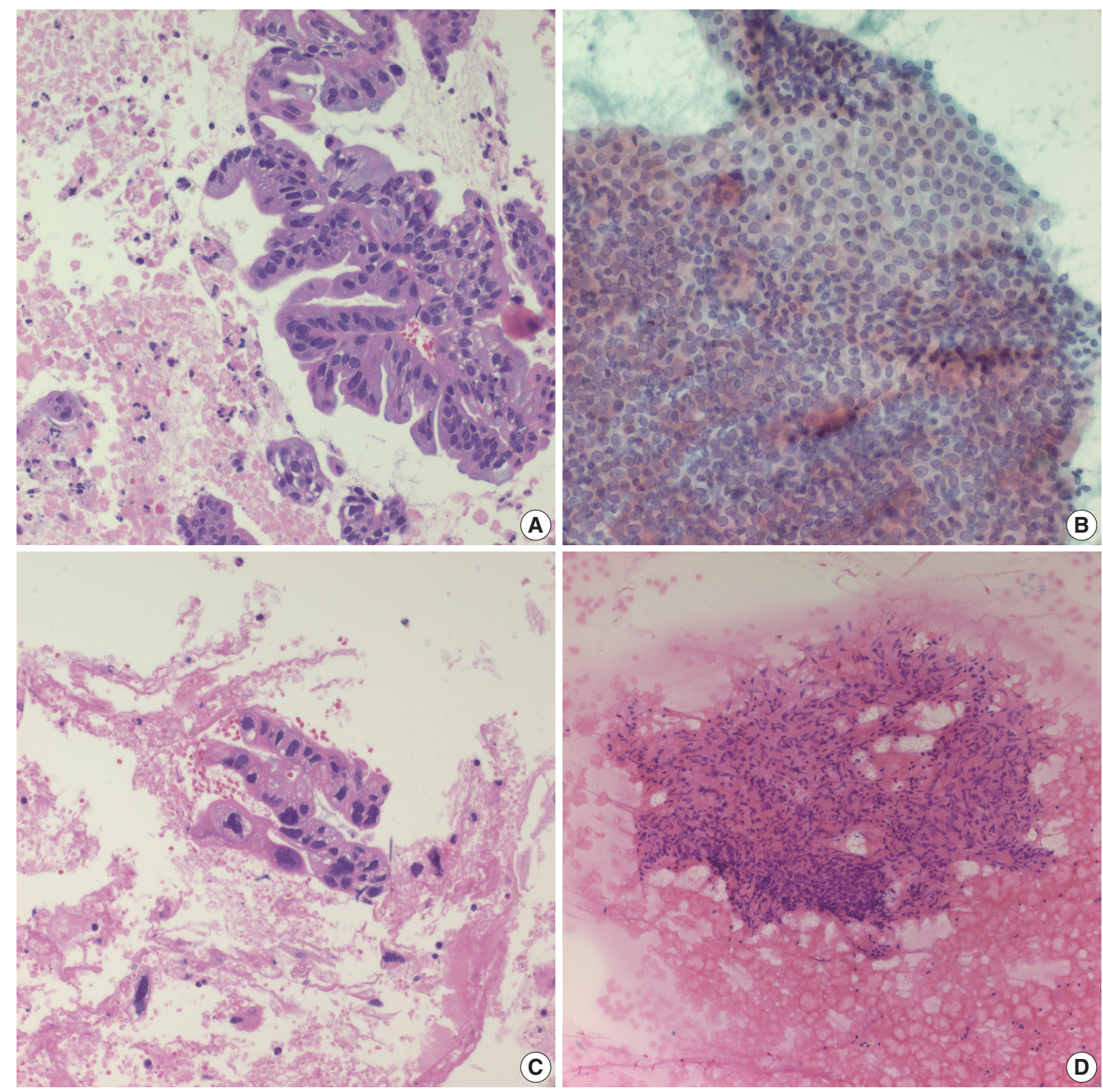

Fig. 2. (A) Ductal adenocarcinoma showing scattered atypical cell nests with complex papillary architecture and necrotic background on biopsy slide. (B) Well differentiated ductal adenocarcinoma cell nest, mimicking normal mucosa, is more hypercellular on conventional cytology smear (Pap). (C) A few atypical tumor cells in a fibrinous background on biopsy slide. Although it does not include necrotic tumor cell debris, the cytologic atypia is sufficient for a diagnosis of malignancy, especially when clinical or radiologic evaluation strongly suggests a malignancy. (D) Tuberculous inflammation shows aggregates of granuloma without viable tumor cells in conventional cytology smear.

pancreas or retroperitoneal region. Due to the high prevalence of tuberculosis in Korea, the possibility of tuberculous lymphadenitis should be considered, especially in EUS-FNAs from older patients. Special stains for acid-fast bacilli and molecular studies can be performed with biopsies or cell blocks to confirm the diagnosis of tuberculosis. Diffuse necrotic backgrounds are not limited to ductal carcinoma or tuberculosis. Necrotic cells may also be seen in EUS-FNA cytology/biopsy of solid pseudopapillary neoplasms (SPNs) and poorly differentiated neuroendocrine carcinomas. However, SPN and neuroendocrine carcinoma are usually accompanied by hypercellular components on cytologic smears, and these neoplasms are relatively easily differentiated on radiologic evaluation. The main differential diagnosis points are summarized in Table 1.

\section{Intraductal papillary mucinous neoplasm}

IPMN is one of the most common pancreatic cystic neoplasms, and EUS-FNA is frequently performed to evaluate the grade of cytologic atypia and presence of an associated invasive carcinoma. It is not uncommon to find mucinous content in cytologic smears from EUS-FNA. Although mucin may be found in EUSFNA material from IPMN, it is not a specific finding. When a mucinous background is noted on cytology/biopsy slides (Fig. $3 \mathrm{~A}$ ), the pathologist should check the radiology findings. If the 
radiologic interpretation strongly suggests IPMN, the pathologist may suggest IPMN in the cytopathologic diagnosis of EUSFNA. However, diagnosis of IPMN on EUS-FNA should be avoided if the radiologic and EUS findings do not suggest IPMN, as the radiologic findings of IPMN are relatively unique. In addition, it is relatively uncommon to observe mucin in cytology slides and/or biopsies from IPMN, especially in LBC. The appearance of aspirated mucin also depends on its vis-

Table 1. Differential diagnosis in EUS-FNA of common pancreatic neoplasms

\begin{tabular}{lcccc}
\hline & DAC & IPMN & NET & SPN \\
\hline Cytologic atypia &,,++++++ & + & + &,$+++^{\mathrm{a}}$ \\
Discohesive pattern & $+^{\mathrm{b}}$ & - &,$+++++^{\mathrm{c}}$ &,$+++++^{\mathrm{d}}$ \\
Organoid cell nest &,+++ & + & - & - \\
Necrotic bakcground &,,$++++++^{\mathrm{e}}$ & - & - & $+^{\mathrm{f}}$ \\
Mucin & $+^{\mathrm{g}}$ &,$+++^{\mathrm{h}}$ & - & - \\
\hline
\end{tabular}

EUS-FNA, endoscopic ultrasound-guided fine needle aspiration; DAC, ductal adenocarcinoma; IPMN, intraductal papillary-mucinous neoplasm; NET, neuroendocrine tumor; SPN, solid pseudopapillary neoplasm.

aSome cases of SPN may show scattered large bizarre nuclei, which correspond to degenerative atypia and do not indicate high-grade malignancy; ' ${ }^{\text {Dis- }}$ cohesive atypical cells may be present in the background of tumor necrosis; 'NET usually has a hypercellular smear with diffuse discohesive pattern and mild cytologic atypia; 'SPNN frequently exhibits plasmacytoid features and occasional clear cytoplasm; 'DAC exhibits a necrotic background composed of necrotic tumor cells and inflammatory cells; 'SPN may show necrotic cells that are mostly hemorrhagic, which is different from tumor necrosis of DAC; ${ }^{9} \mathrm{~A}$ mucinous component may be present in EUS-FNA cytology/biopsy from DAC. However, it is frequently mixed with a necrotic background and atypical tumor cells; "IPMN may have a diffuse mucinous background. However, it depends upon the viscosity of mucin.
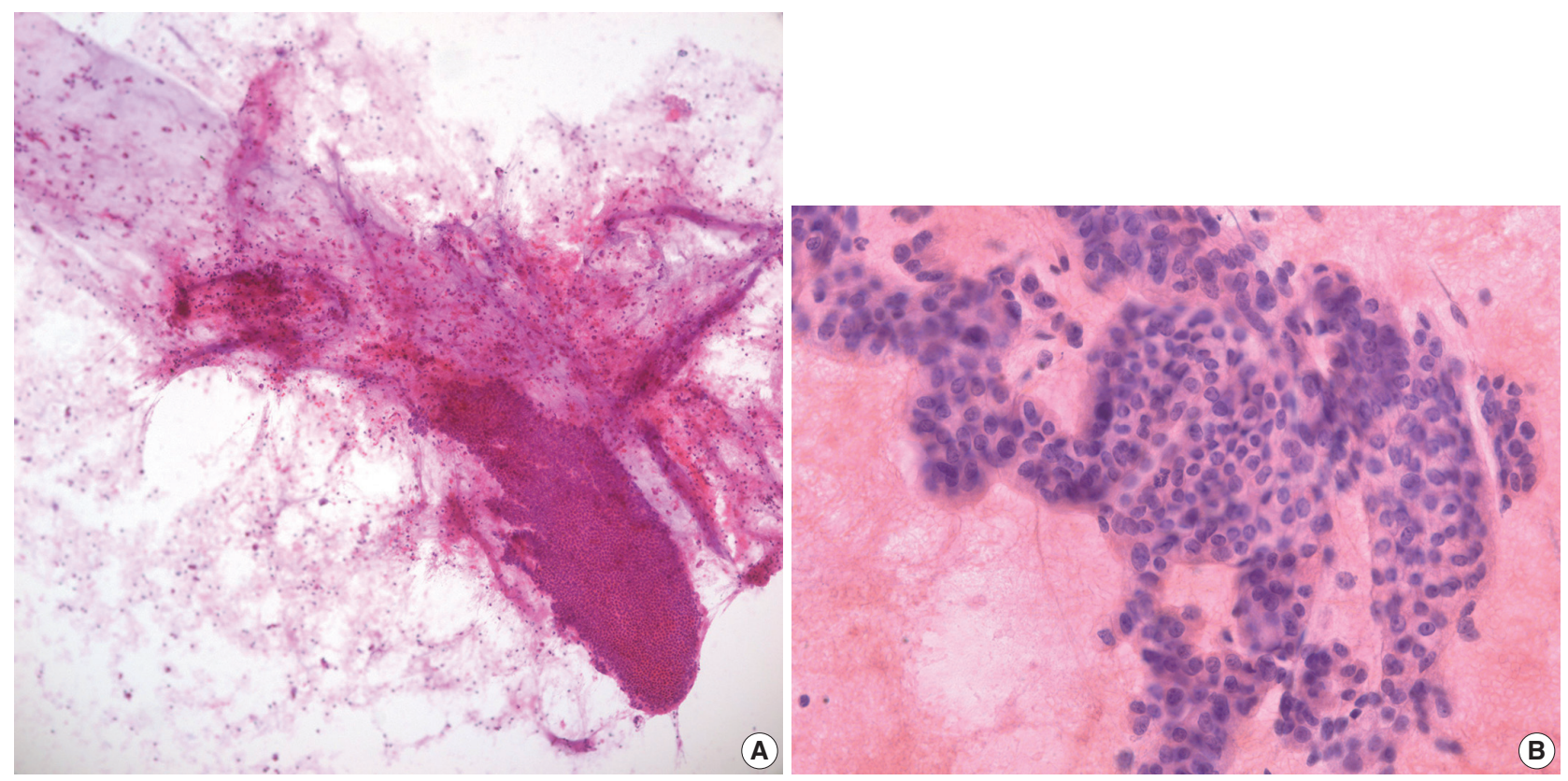

cosity. Main duct type IPMNs with intestinal phenotype usually show viscous mucin, similar to that seen in appendiceal mucinous neoplasms. Conversely, branch duct type IPMNs contain relatively less viscous gastric foveolar-type mucin, resulting in a more transparent mucinous background that is difficult to recognize on cytologic smears. The typical cytologic features of IPMNs include papillary clusters of columnar epithelial cells containing cytoplasmic mucin (Fig. 3B), and IPMNs may be out intraductal or intracystic papillary lesions may not demonstrate typical papillary epithelial cell nests on EUS-FNA slides. Sometimes it is difficult to differentiate papillary cell nests from contaminated intestinal mucosa. If the IPMN is branch duct type with gastric foveolar type epithelium, it may be difficult to differentiate between mucinous epithelium of the IPMN and contaminated gastric mucosa. Pathologists should assess the radiologic findings of IPMN. If there are no mural or papillary nodules of IPMN on radiologic evaluation, the cellular nest of epithelial mucosa probably corresponds to contaminated gastric mucosa, not the epithelial component of IPMN. The presence of scattered goblet cells within monotonous-looking cell nests is a helpful clue that can differentiate normal intestinal mucosa from IPMNs (Fig. 1B). It is therefore important that pathologists assess the anatomic location of target lesions on clinical EUS-FNA reports; lesions in the pancreatic head and uncinate process are usually targeted via the duodenal wall, while those graded according to the degree of nuclear atypia. IPMNs with-

Fig. 3. Intraductal papillary mucinous neoplasm. (A) A mucinous background is seen on conventional cytology smear. (B) A papillary epithelial nest showing high-grade dysplasia on conventional cytology smear. 
located in the body and tail are usually accessed via the gastric wall. If pathologists identify small intestinal mucosa-like epithelial cell nests in EUS-FNA from the pancreas body or tail with a clinical impression of main duct type IPMN, it likely corresponds to the epithelial component of IPMN because the EUS-FNA needle penetrated the gastric wall, not the duodenal mucosa. However, if radiologic findings indicate branch duct type IPMN, which is mostly composed of gastric phenotype epithelium, it may be difficult to discriminate between contaminated gastric mucosa and the epithelial component of gastric phenotype IPMN. The papillary nests within cystic lesions are relatively small in branch duct type IPMN. If a pathologist finds relatively large nests of intestinal mucosa, it may corresponds to contaminant intestinal mucosa rather than papillary nests of branch duct type IPMN.

\section{Serous/mucinous cystic neoplasm}

Serous cystic neoplasms (SCNs) are not infrequently submitted for EUS-FNA evaluation. It is uncommon to observe the typical serous lining cells on cytology smears and/or biopsy, especially in aspirates from unilocular or macrocystic lesions. However, if the SCN is a multilocular cystic lesion, some serous epithelial cell clusters composed of cuboidal cells with clear cytoplasm may be seen in cytology and/or biopsy slides. If the pathologist can find scattered subepithelial capillaries in the biopsy slide, it is supportive of an SCN. In most cases, SCN is easily detected by radiologic evaluation, except for macrocystic SCN. Radiologic correlation is essential in pathologic diagnosis of SCN in EUSFNA.

Mucinous cystic neoplasm (MCN) is a relatively uncommon cystic neoplasm of the pancreas. Mucinous epithelial lining and an underlying ovarian-type stroma are characteristic histologic findings. However, it may be difficult to observe wellpreserved mucinous epithelium and ovarian-type stroma, even in resected specimens. It is even harder to identify mucinous epithelial cells and/or ovarian-type stroma in EUS-FNA cytology/biopsy. If some mucinous epithelial cells are seen, the pathologist should check the clinical findings and radiologic interpretation. The characteristic clinical setting is a middleaged female with a pancreatic unilocular cystic neoplasm or multilocular septated cyst without ductal communication. Invasive carcinomas may arise from MCNs, and the biologic behavior of $\mathrm{MCN}$-associated invasive carcinomas is aggressive even at an early stage. It may be difficult to detect early invasion by EUS-FNA, as these early invasive components do not create mass lesions on radiologic or EUS findings.

\section{Neuroendocrine neoplasms}

Aspirated material from pancreatic neuroendocrine neoplasms usually results in a hypercellular smear. Although some highgrade neuroendocrine carcinomas have an accompanying necrotic background, well-differentiated pancreatic neuroendocrine tumors (NETs) rarely show necrosis on cytology smears. Pancreatic NET is classified by a 3-tier scheme [10]. Grade 1 and 2 NETs are well-differentiated neuroendocrine tumors with mitotic rate of $<2 / 2 \mathrm{~mm}^{2}$ or $\mathrm{Ki}-67$ index $<3 \%$ and mitotic rate of $2-20 / 2 \mathrm{~mm}^{2}$ or Ki-67 index $3 \%-20 \%$, respectively. Well-differentiated grade 3 NETs show a mitotic rate of $>20 / 2 \mathrm{~mm}^{2}$ or Ki-67 index $>20 \%$. Tumor cells from well-differentiated NETs usually show mild cytologic atypia, sometimes with characteristic salt-and-pepper chromatin pattern, which is more frequently found on cytology (Fig. 4A). NET cells on biopsy slides are more discohesive epithelial cells with round to vesicular nuclei and plump cytoplasm (Fig. 4B). While NETs usually demonstrate scant cytoplasm, some NETs may have relatively abundant cytoplasm (Fig. 4C). In such cases, it may be difficult to distinguish NET from SPN. The cytologic findings of discohesive cells and plasmacytoid features suggest the diagnosis of SPN. Immunostaining for $\beta$-catenin is a useful and reliable differential diagnostic tool, as other neuroendocrine markers (e.g., CD56, synaptophysin) can be expressed in both tumors [11]. NETs usually express $\beta$-catenin with a membranous pattern whereas SPNs exhibit nuclear $\beta$-catenin staining. Pathologists should correlate with radiologic findings before performing immunohistochemical stains. NETs are usually well demarcated hyper-enhancing masses. If a pancreatic mass is partially solid and cystic on radiologic evaluation, it is more likely to be an SPN than a NET. Grading of NET on EUS-FNA biopsies is well correlated with that of surgically resected tumors $[12,13]$. However, considering the uneven distribution of $\mathrm{Ki}-67$ hot spots in tissue sections, it is still possible that the $\mathrm{Ki}-67$ labeling index of a biopsy does not represent the proliferation index of the whole tumor [14]. Aspirates from high-grade small cell neuroendocrine carcinomas demonstrate hyperchromatic small cell carcinomalike features with or without a necrotic background (Fig. 4D). Differential diagnosis of metastatic lung small cell from may be difficult because the cytologic findings are similar in both lesions. Ancillary immunostaining for thyroid transcription factor 1 (TTF-1) may be helpful, as metastatic small cell carcinoma from the lung usually expresses TTF-1 immunoreactivity. However, pathologists should also correlate with the clinical history and radiologic findings for systemic metastasis. If patients have a past medical history of lung cancer with multiple metastasis, 

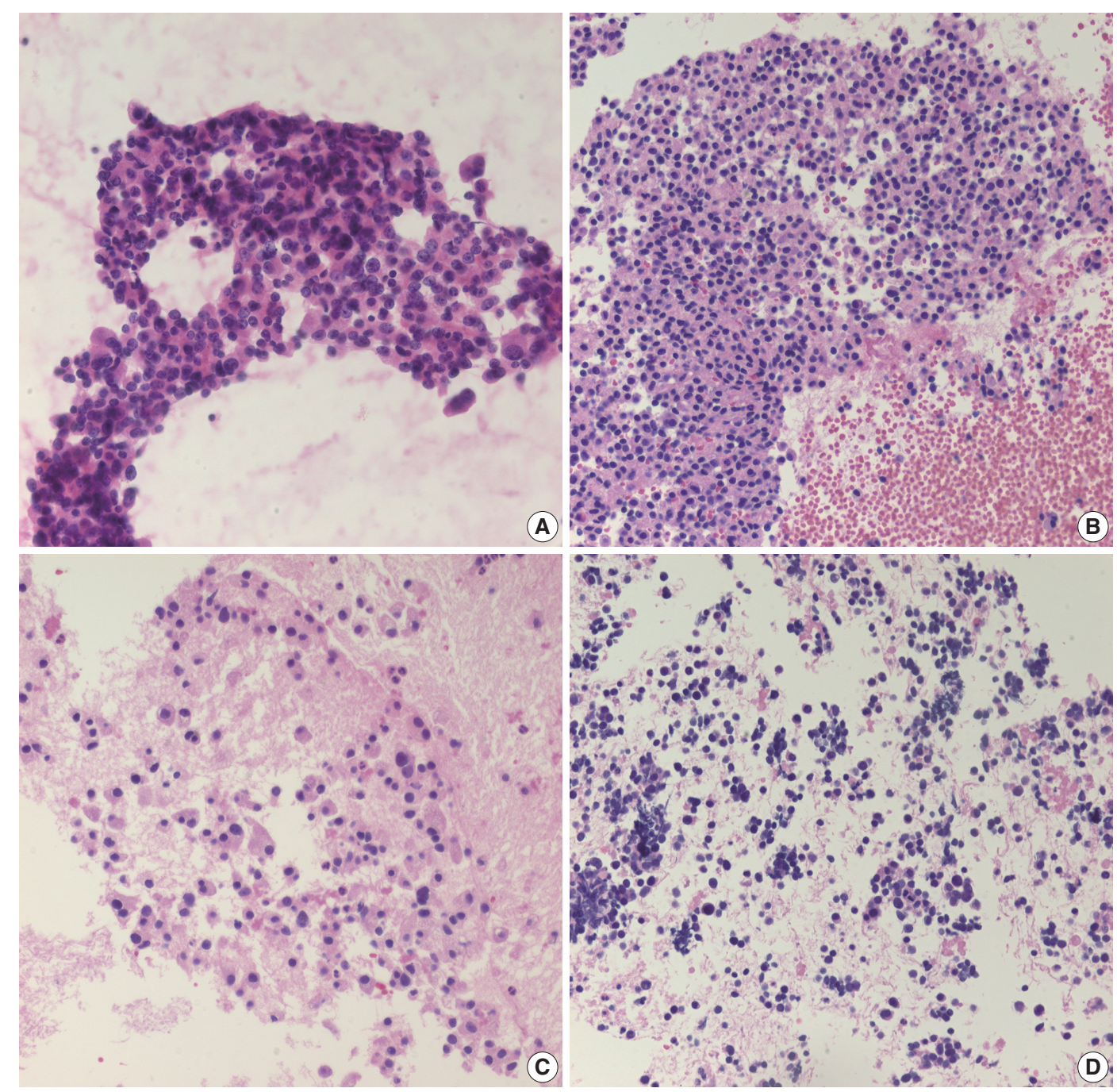

Fig. 4. (A) Well-differentiated neuroendocrine tumor cells show the characteristic salt-and-pepper chromatin pattern on conventional cytology smear. (B) On biopsy, well-differentiated neuroendocrine tumor cells show diffuse nest-like arrangement of monotonous nuclei with mild atypia. (C) Scattered epithelioid cells with hyperchromatic nuclei and abundant cytoplasm may be seen in well-differentiated neuroendocrine tumors. (D) Poorly differentiated neuroendocrine carcinoma shows scattered hyperchromatic nuclei.

it should be easily suspected regardless of ancillary test results.

\section{Solid pseudopapillary neoplasm}

The characteristic clinical setting of SPN is a middle age female with a pancreatic mass in a distal pancreas location, either the body or tail. Although exceptional cases may occur, pathologists should assess these two clinical findings when diagnosing SPN. Radiologic examination typically shows a solid and cystic tumor with or without necrotic contents. However, SPNs smaller than $2 \mathrm{~cm}$ may present as solid tumors and may be interpreted as NETs on radiologic evaluation. Cytologic smears of SPN are usually discohesive and hypercellular, and the cells demonstrate mild nuclear atypia and plasma cell-like features (Fig. 5A).
The presence of myxoid pseudopapillae is a characteristic pathologic finding of SPN. However, it is relatively uncommon to see the typical myxoid pseudopapillary pattern of SPN in cytologic smears of EUS-FNA (Fig. 5B). Scattered pleomorphic or bizarre cells from SPN are reported to reflect degenerative change rather than high-grade malignancy, which may be overestimated on EUS-FNA pathologic diagnosis [15]. Sometimes, tumor cells of SPN show clear cell change that mimics renal cell carcinoma. Pathologists should correlate with the patient's past medical history before confirming a diagnosis of metastatic renal cell carcinoma to the pancreas. 

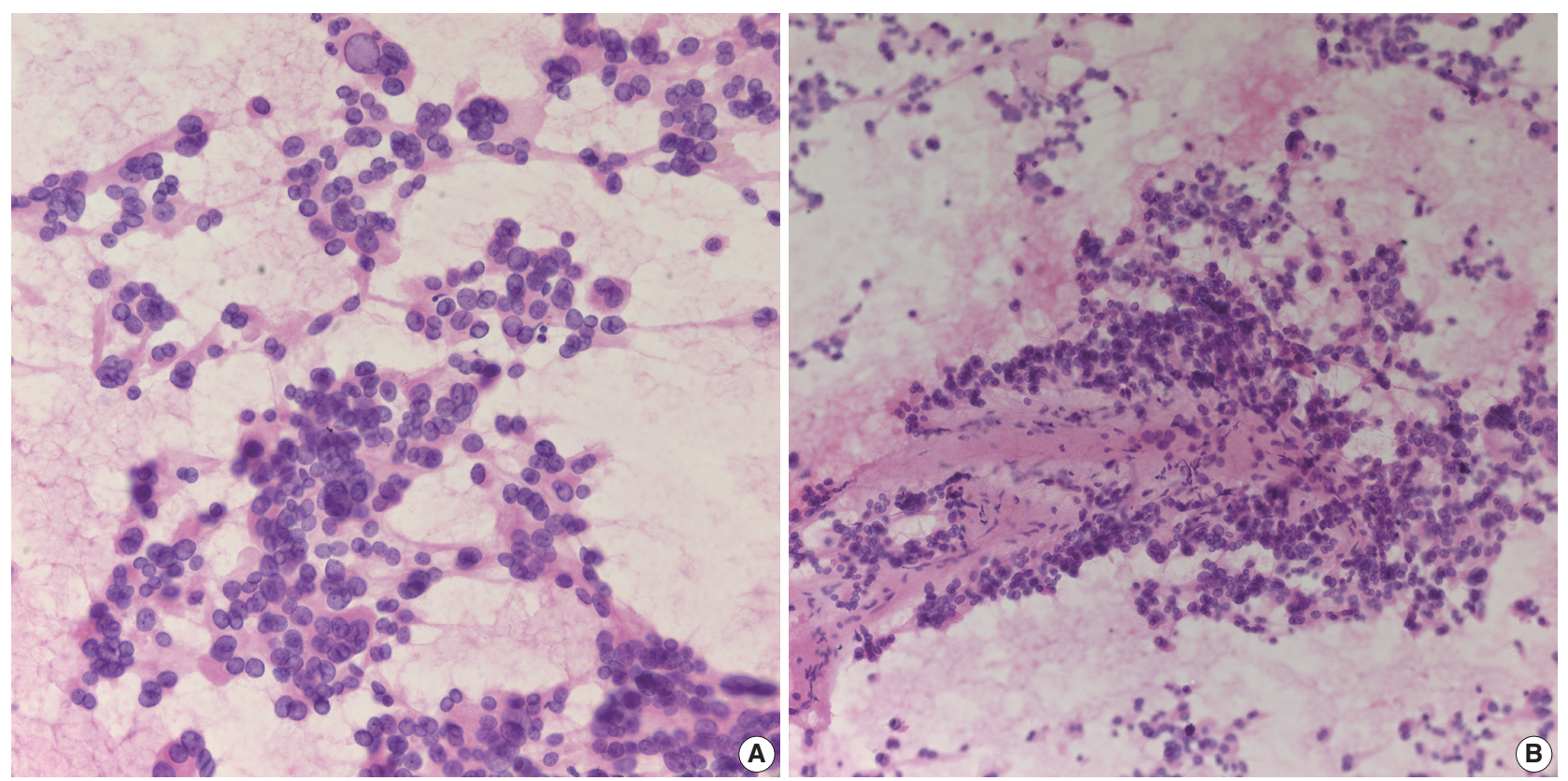

Fig. 5. (A) Scattered solid pseudopapillary neoplasm cells show discohesive organoid pattern with vesicular nuclei. (B) Solid pseudopapillary neoplasms show myxoid pseudopapillae with scattered plasmacytoid cells.

\section{Autoimmune disease}

EUS-FNA is rarely performed to evaluate autoimmune disease that involves the pancreas or bile duct. If the clinical history or radiologic findings are characteristic, the pathologic findings of EUS-FNA biopsy may provide typical histologic findings of dense lymphoplasmacytic infiltration and/or storiform fibrosis. However, if the patient history or radiologic findings are vague, it may be difficult to differentiate conventional chronic pancreatitis from autoimmune pancreatitis or cholangitis. Especially when presenting as a local mass-forming lesion, it is very difficult to suspect autoimmune disease if EUS-FNA cytology/biopsy provides only surface mucosa [16]. It should be emphasized that the pathologic diagnosis of autoimmune disease should be made after excluding malignancy in the differential diagnosis. IgG4 immunostaining is essential in evaluation of IgG4-related autoimmune disease that involves the pancreas or bile duct. If IgG4 positivity is sufficient by immunostain study, IgG4-related autoimmune disease can be diagnosed. However, pathologists should be careful to exclude autoimmune disease with insufficient IgG4 positivity because increased IgG4-positive plasma cells can be found in other inflammatory conditions or even in some malignancies [17].

\section{Metastatic tumor}

Some malignant tumors may metastasize to the pancreas; renal cell carcinoma is one of the most common metastatic tumors in the pancreas. However, it is uncommon for pathologists to see metastatic tumors on EUS-FNA cytology and/or biopsy because it is difficult to evaluate metastasis on pancreas EUSFNA. It is challenging to consider metastatic renal cell carcinoma without pertinent clinical information. Metastatic renal cell carcinoma sometimes does not exhibit characteristic clear cell features on EUS-FNA cytology and/or biopsy specimens, instead showing monotonous cell nests with abundant capillaries (Fig. 6A). Metastatic small cell carcinoma from the lung is difficult to differentiate from primary poorly differentiated neuroendocrine carcinoma of the pancreas because the pathologic findings are very similar (Fig. 6B). Immunostain for TTF-1 and correlation with clinical history are essential for differential diagnosis of metastasis.

\section{WHAT ANCILLARY TESTS CAN BE APPLIED TO ENDOSCOPIC ULTRASOUND-GUIDED FINE NEEDLE ASPIRATION?}

Many ancillary tests can be applied for pathologic evaluation of EUS-FNA specimens. Immunohistochemistry on biopsy specimens or cell blocks is the most widely used method. Immunohistochemical stains are particularly helpful in the differential diagnosis of NETs (e.g., CD56, synaptophysin, chromogranin), SPNs (e.g., nuclear $\beta$-catenin, CD10), acinar cell neoplasms (e.g., trypsin, chymotrypsin, BCL10), and various metastatic tumors $[11,18,19]$. In addition, Ki-67 labeling indi- 

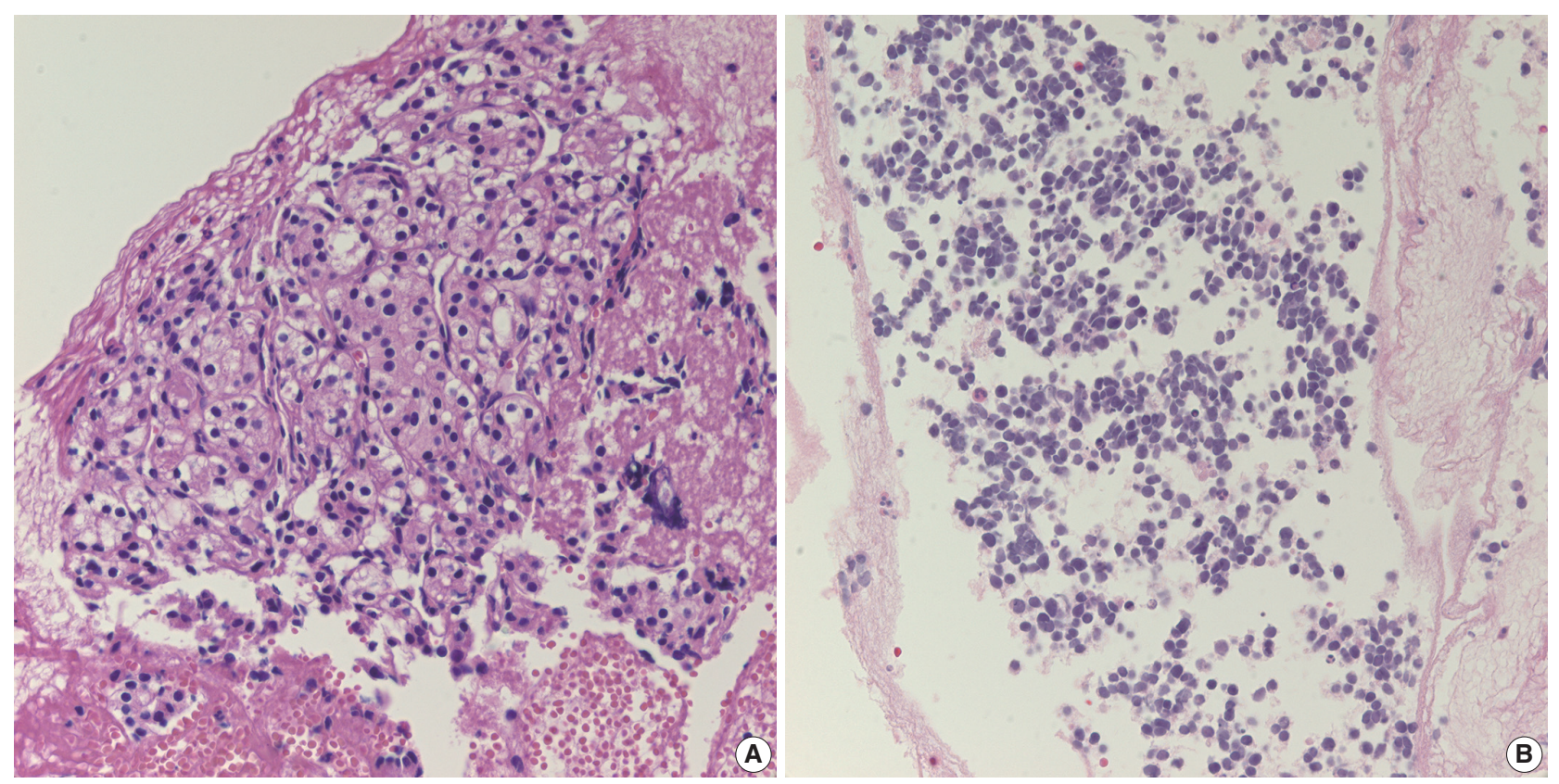

Fig. 6. (A) Metastatic renal cell carcinoma shows clear to granular cytoplasm on endoscopic ultrasound-guided fine needle aspiration (EUSFNA) biopsy. (B) Metastatic small cell carcinoma from the lung exhibited discohesive small round cells with molding pattern on EUS-FNA biopsy.

ces may be evaluated to grade NETs in EUS-FNA aspirates. Although less frequently performed to diagnose ductal adenocarcinomas, immunohistochemical stains for $\mathrm{p} 53$ (overexpression or complete loss of expression), SMAD4 (loss of expression), and carcinoembryonic antigen (cytoplasmic expression) are useful in the differential diagnosis between ductal adenocarcinomas and benign reactive epithelial cells $[11,20]$. Fluorescence in situ hybridization for loss or alterations in copy number of $9 \mathrm{p} 21$, 3, 7, and 17 improves sensitivity for diagnosing ductal adenocarcinoma [21]. DNA sequencing, including next-generation sequencing, may also have a role in EUS-FNA diagnosis in the future. For example, testing for KRAS and GNAS mutations would help to discriminate between IPMN (KRAS or GNAS mutations in >96\%), MCN (KRAS mutations but no GNAS mutations), and non-neoplastic cysts (e.g., pseudocyst), especially when there is only a scant amount of cells in the aspirated cyst fluid $[11,22,23]$. Molecular tests can be applied for differential diagnosis in pancreatic cystic fluid [24]. Some molecular panels are reportedly predictive in differential diagnosis of pancreatic cystic neoplasms. Furthermore, next-generation sequencing can be used in preoperative pathologic differential diagnosis of pancreas cystic lesions [25]. Molecular tests may be more widely used in pathologic evaluation of pancreas cystic lesions, which provide a very limited cellular component. Samples from EUS-FNA showed superior DNA quality than formalin-fixed paraffin-embedded tissue in some molecular analysis
[26]. However, any molecular test alone does not guarantee specific pathologic diagnosis, and they are more useful when used as supportive evidence rather than direct evidence of a specific disease entity. Practically, it is not uncommon to experience histopathological and immunohistochemical discrepancies between cytology/biopsy and resection specimens $[27,28]$. False negative results are more common on initial cytology/biopsy samples due to the heterogeneous histopathology and genomic profiles [29,30].

Clinical applications of molecular testing in practical pathologic diagnosis are limited because medical insurance reimbursement for molecular tests from EUS-FNA of various pancreatic lesions is not yet approved in Korea. Practical ancillary tests for EUS-FNA specimens in most Korean institutions are limited to immunohistochemical stains. However, when unresectable pancreatic cancer is being considered, more ancillary tests may be used because the EUS-FNA specimen is the only sample available to evaluate histopathologic and molecular characteristics of the tumor and provide information for precision medicine therapy.

\section{HOW SHOULD PATHOLOGISTS CORRELATE CLINICAL AND/OR RADIOLOGIC FINDINGS WHEN INTERPRETING ENDOSCOPIC ULTRASOUND-GUIDED FINE NEEDLE ASPIRATION SPECIMENS?}

EUS-FNA cytology/biopsy slides commonly demonstrate only 
a few atypical cells that may be insufficient for a conclusive diagnosis of a malignancy. Therefore, it is often difficult for general surgical pathologists to confidently make a conclusive diagnosis on EUS-FNA specimens. In these cases, it may be better to adjust the threshold of malignancy according to the clinical and radiologic findings. For example, if a patient has multiple liver metastases and peritoneal seeding, the pathologist can suggest "malignancy" by lowering the threshold of malignancy, even with a few atypical cells on EUS-FNA. However, if the same cytology and/or biopsy is encountered in the setting of a clinical impression of "rule out autoimmune pancreatitis vs. hidden malignancy," it may be better to provide a diagnosis of "atypical cells, uncertain malignant potential or cannot exclude malignancy." Pathologists should raise their threshold of malignancy in such situations. Although this may be criticized as unprofessional, pathologists should be flexible to enable better communication with clinicians and improve patient management in diagnosis of EUS-FNA for pancreatic lesions. Clinical-pathologic correlation is essential in pathologic diagnosis of EUS-FNA from pancreatic lesions. If an EUS-FNA does not provide any atypical cells in a clinical setting of malignancy, pathologists should suggest the possibility of a non-representative sample in the pathology report. Suggested terminology is: "The possibility of non-representative sample remains. Re-aspiration is recommended to rule out malignancy." Many clinicians think that a pathologic diagnosis of malignancy is difficult on EUS-FNA specimens. However, pathologic confirmation of a true negative is more difficult than confirmation of a true positive. Pathologists should be aware of inter-observer disagreement in radiologic interpretation of various pancreatic lesions. Due to the deep location of the pancreas, it is not feasible to obtain as many tumor cells as in cytology smears of the thyroid, breast, and uterine cervix. An open biopsy in the operation room would be required to obtain such quantities of tumor cells, which would be a burden to patients. In the era of molecular testing for precision medicine therapy, tumor volume is certainly an important issue. Given the small amounts of tumor cells obtained by EUS-FNA, more reliable sensitive molecular assays should be developed, such as circulating tumor DNA or mutant KRAS in circulating exosome [31,32]. Although there have been some advances in precision medicine strategies for pancreas cancer, there are currently few targets for precision medicine in pancreatic tumors [33,34]. Until now, the main goal of the EUS-FNA cytology/biopsy is confirmation of a malignancy or non-neoplastic disease to guide decisions for the next step in therapy and patient management.

\section{CONCLUSION}

EUS-FNA cytology/biopsy for pancreatic lesions has become more routine in daily practice. Pathologists should be familiar with clinical and/or radiologic findings to make an accurate pathologic diagnosis of pancreatic lesions. Scant cellular smears or marked degeneration artifact are more likely from EUS-FNA for pancreatic lesions than for specimens of another organs. Pathologists should adjust their threshold of malignancy according to the clinical situation to avoid over- or under-treatment. Ancillary tests, including molecular analysis, can be helpful in differential diagnosis of EUS-FNA for various pancreatic lesions. However, they should be used as supportive evidence rather than direct diagnostic evidence in pathologic diagnosis of EUS-FNA.

\section{Ethics Statement}

Not applicable.

\section{ORCID}

Haeryoung Kim https://orcid.org/0000-0002-4205-9081

Kee-Taek Jang https://orcid.org/0000-0001-7987-4437

\section{Author Contributions}

Conceptualization: KTJ. Writing-original draft: HK, KTJ. Writing-review \& editing: HK, KTJ. Approval of final manuscript: all authors.

\section{Conflicts of Interest}

H.K., a contributing editor of the Journal of Pathology and Translational Medicine, was not involved in the editorial evaluation or decision to publish this article. All remaining authors have declared no conflicts of interest.

\section{Funding Statement}

No funding to declare.

\section{References}

1. DiMagno EP, Buxton JL, Regan PT, et al. Ultrasonic endoscope. Lancet 1980; 1: 629-31.

2. Vilmann P, Jacobsen GK, Henriksen FW, Hancke S. Endoscopic ultrasonography with guided fine needle aspiration biopsy in pancreatic disease. Gastrointest Endosc 1992; 38: 172-3.

3. Chun JW, Lee K, Lee SH, et al. Comparison of liquid-based cytology with conventional smear cytology for EUS-guided FNA of solid pancreatic masses: a prospective randomized noninferiority study. Gastrointest Endosc 2020; 91: 837-46.

4. Mitoro A, Nishikawa T, Yoshida M, et al. Diagnostic efficacy of liquid-based cytology in endoscopic ultrasound-guided fine needle aspiration for pancreatic mass lesions during the learning curve: a retrospective study. Pancreas 2019; 48: 686-9.

5. Yung RC, Otell S, Illei P, et al. Improvement of cellularity on cell block preparations using the so-called tissue coagulum clot method during endobronchial ultrasound-guided transbronchial fine-needle aspiration. Cancer Cytopathol 2012; 120: 185-95. 
6. Lee KS, Sekhar A, Rofsky NM, Pedrosa I. Prevalence of incidental pancreatic cysts in the adult population on MR imaging. Am J Gastroenterol 2010; 105: 2079-84.

7. Wittmann J, Kocjan G, Sgouros SN, Deheragoda M, Pereira SP. Endoscopic ultrasound-guided tissue sampling by combined fine needle aspiration and trucut needle biopsy: a prospective study. Cytopathology 2006; 17: 27-33.

8. Lee YN, Moon JH, Choi HJ, et al. Tissue acquisition for diagnosis of biliary strictures using peroral cholangioscopy or endoscopic ultrasound-guided fine-needle aspiration. Endoscopy 2019; 51: 50-9.

9. Lee JK, Lee KT, Choi ER, et al. A prospective, randomized trial comparing 25-gauge and 22-gauge needles for endoscopic ultrasoundguided fine needle aspiration of pancreatic masses. Scand J Gastroenterol 2013; 48: 752-7.

10. Rindi G, Klimstra DS, Abedi-Ardekani B, et al. A common classification framework for neuroendocrine neoplasms: an International Agency for Research on Cancer (IARC) and World Health Organization (WHO) expert consensus proposal. Mod Pathol 2018; 31: $1770-86$

11. Misdraji J, Centeno BA, Pitman MB. Ancillary tests in the diagnosis of liver and pancreatic neoplasms. Cancer Cytopathol 2018; 126 Suppl 8: 672-90.

12. Li J, Lin JP, Shi LH, et al. How reliable is the Ki-67 cytological index in grading pancreatic neuroendocrine tumors? A meta-analysis. J Dig Dis 2016; 17: 95-103.

13. Weynand B, Borbath I, Bernard V, et al. Pancreatic neuroendocrine tumour grading on endoscopic ultrasound-guided fine needle aspiration: high reproducibility and inter-observer agreement of the Ki-67 labelling index. Cytopathology 2014; 25: 389-95.

14. Hwang HS, Kim Y, An S, et al. Grading by the Ki-67 labeling index of endoscopic ultrasound-guided fine needle aspiration biopsy specimens of pancreatic neuroendocrine tumors can be underestimated. Pancreas 2018; 47: 1296-303.

15. Kim SA, Kim MS, Kim MS, et al. Pleomorphic solid pseudopapillary neoplasm of the pancreas: degenerative change rather than high-grade malignant potential. Hum Pathol 2014; 45: 166-74.

16. Kim S, Bae H, Choi M, et al. Isolated mass-forming IgG4-related cholangitis as an initial clinical presentation of systemic IgG4-related disease. J Pathol Transl Med 2016; 50: 300-5.

17. Lee HE, Zhang L. Immunoglobulin G4-related hepatobiliary disease. Semin Diagn Pathol 2019; 36: 423-33.

18. Heymann JJ, Siddiqui MT. Ancillary techniques in cytologic specimens obtained from solid lesions of the pancreas: a review. Acta Cytol 2020; 64: 103-23.

19. Layfield LJ, Ehya H, Filie AC, et al. Utilization of ancillary studies in the cytologic diagnosis of biliary and pancreatic lesions: the Papanicolaou Society of Cytopathology guidelines for pancreatobiliary cytology. Diagn Cytopathol 2014; 42: 351-62.
20. Soyer OM, Baran B, Ormeci AC, et al. Role of biochemistry and cytological analysis of cyst fluid for the differential diagnosis of pancreatic cysts: a retrospective cohort study. Medicine (Baltimore) 2017; 96: e5513.

21. Kubiliun N, Ribeiro A, Fan YS, et al. EUS-FNA with rescue fluorescence in situ hybridization for the diagnosis of pancreatic carcinoma in patients with inconclusive on-site cytopathology results. Gastrointest Endosc 2011; 74: 541-7.

22. Wu J, Matthaei H, Maitra A, et al. Recurrent GNAS mutations define an unexpected pathway for pancreatic cyst development. Sci Transl Med 2011; 3: 92ra66.

23. Jones M, Zheng Z, Wang J, et al. Impact of next-generation sequencing on the clinical diagnosis of pancreatic cysts. Gastrointest Endosc 2016; 83: 140-8.

24. Ngamruengphong S, Lennon AM. Analysis of pancreatic cyst fluid. Surg Pathol Clin 2016; 9: 677-84.

25. Rosenbaum MW, Jones M, Dudley JC, Le LP, Iafrate AJ, Pitman MB. Next-generation sequencing adds value to the preoperative diagnosis of pancreatic cysts. Cancer Cytopathol 2017; 125: 41-7.

26. Hartley CP, Mahajan AM, Selvaggi SM, Rehrauer WM. FNA smears of pancreatic ductal adenocarcinoma are superior to formalin-fixed paraffin-embedded tissue as a source of DNA: Comparison of targeted KRAS amplification and genotyping in matched preresection and postresection samples. Cancer Cytopathol 2017; 125: 838-47.

27. Park YJ, Kim GH, Park DY, et al. Histopathologic discrepancies between endoscopic forceps biopsy and endoscopic resection specimens in superficial esophageal squamous neoplasms. J Gastroenterol Hepatol 2019; 34: 1058-65.

28. Ilie M, Long-Mira E, Bence C, et al. Comparative study of the PDL1 status between surgically resected specimens and matched biopsies of NSCLC patients reveal major discordances: a potential issue for anti-PD-L1 therapeutic strategies. Ann Oncol 2016; 27: 147-53.

29. Cros J, Raffenne J, Couvelard A, Pote N. Tumor heterogeneity in pancreatic adenocarcinoma. Pathobiology 2018; 85: 64-71.

30. Prasetyanti PR, Medema JP. Intra-tumor heterogeneity from a cancer stem cell perspective. Mol Cancer 2017; 16: 41.

31. Allenson K, Castillo J, San Lucas FA, et al. High prevalence of mutant KRAS in circulating exosome-derived DNA from early-stage pancreatic cancer patients. Ann Oncol 2017; 28: 741-7.

32. Takai E, Totoki Y, Nakamura H, Kato M, Shibata T, Yachida S. Clinical utility of circulating tumor DNA for molecular assessment and precision medicine in pancreatic cancer. Adv Exp Med Biol 2016; 924: 13-7.

33. Torres C, Grippo PJ. Pancreatic cancer subtypes: a roadmap for precision medicine. Ann Med 2018; 50: 277-87.

34. Chantrill LA, Nagrial AM, Watson C, et al. Precision medicine for advanced pancreas cancer: the Individualized Molecular Pancreatic Cancer Therapy (IMPaCT) trial. Clin Cancer Res 2015; 21: 2029-37. 